\title{
Color pattern recognition with circular component whitening
}

\author{
I. Moreno, V. Kober, V. Lashin, and J. Campos \\ Department of Physics, Autonomous University of Barcelona, E-08193 Bellaterra, Spain \\ L. P. Yaroslavsky \\ Department of Interdisciplinary Studies, Tel Aviv University, Tel Aviv 69978, Israel \\ M. J. Yzuel \\ Department of Physics, Autonomous University of Barcelona, E-08193 Bellaterra, Spain
}

Received September 18, 1995

\begin{abstract}
Polychromatic object recognition based on circular whitening preprocessing of red-green-blue components and multichannel matched filtering is described. Computer simulations and experimental results are provided to facilitate recognizing a color target among objects of similar shape but with different color contents. Experimental results are obtained with an optical correlator with two spatial light modulators, one to introduce the scene and the second one to introduce the filter. (C) 1996 Optical Society of America
\end{abstract}

Color pattern recognition based on optical correlation methods has been a subject of intensive investigations in the past few years. ${ }^{1-5}$ In general, a color image provides more information than a monochromatic one, and therefore additional color information could contribute in some way to a better correlation performance. Various approaches considering how to use the color contents of a signal to improve pattern recognition have been suggested. One of the simplest ways to take into account the color content of objects is to carry out correlation filtering in three red-green-blue (RGB) channels independently, then to make either arithmetic or logical elementwise operations over the correlation outputs, and finally to find signal maxima on the resulting correlation plane. Even such a simple approach using a phase-only filter ${ }^{6}$ in the multichannel correlation procedure provides good results that one can use to recognize a target with a given shape and color combinations of an object, regardless of what the combinations are. ${ }^{3-5}$ Color information of a signal can be involved in the recognition process more effectively by introduction of an elementwise proprocessing of color components before optical multichannel correlations. Badiqué $e t a l .^{2}$ have developed a means of projecting polychromatic images on a generalized color plane. This method reduces the number of components used in the correlation process and enhances the ability of the correlator to discriminate between images of different colors. Another fruitful approach involves preprocessing of the RGB components on the basis of human perception. ${ }^{7}$

It is known that the RGB components of the original color image are strongly correlated with one another. We propose to perform component transformation by carrying out whitening operation along the color axis. ${ }^{8}$ The transformed color components can be considered independent components in the above sense, and channel correlations can be performed independently.

The purpose of this paper is to investigate the performance of color pattern recognition based on RGB component preprocessing (whitening) and multichannel correlations. We show that by using a phase-only filter in each transformed channel, and then adding correlation outputs, one can achieve a good recognition performance. In addition, the use of spatial light modulators in correlation architectures permits flexible implementation of correlation experiments with different images and filters.

The whitening of operation applied over a discrete sequence is based on the discrete Fourier transform formalism. Because in this case a discrete sequence is assumed to be periodic, we refer to the whitening operation applied over a color signal as a circular whitening (CW) operation.

Now we consider the CW operation. Let $\left\{s_{n}(x, y)\right\}$ be a color image, for example, $s_{0}(x, y) \equiv R, s_{1}(x, y) \equiv G$, and $s_{2}(x, y) \equiv B$. Here, $x$ and $y$ are the spatial coordinates and $n$ denotes the index of primary color components $(n=0,1,2)$. The transformed color components $\left\{\tilde{s}_{n}(x, y)\right\}$ can be written by use of discrete Fourier transforms as follows:

$$
\tilde{s}_{n}(x, y)=\sum_{k=0}^{2} \tilde{S}_{k}(x, y) \exp (i 2 \pi k n / 3),
$$

where $\left\{\tilde{S}_{k}(x, y)\right\}$ is the discrete spectrum of the transformed color image and $k$ is the index of the spectrum components $(k=0,1,2)$. This discrete spectrum can be expressed by means of the following whitening operation:

$$
\tilde{S}_{k}(x, y)=S_{k}(x, y) /\left|S_{k}(x, y)\right|,
$$

where $\left\{S_{k}(x, y)\right\}$ is the inverse discrete Fourier transform of the input color image along the color axis. Finally, $\tilde{s}_{n}(x, y)$ are obtained directly from Eqs. (1) and (2) and can be written as

$$
\begin{aligned}
\tilde{s}_{n}(x, y)= & \operatorname{sign}\left[s_{0}(x, y)+s_{1}(x, y)+s_{2}(x, y)\right] \\
& +\frac{1}{\Delta}\left[2 s_{n}(x, y)-\sum_{i \neq n} s_{i}(x, y)\right],
\end{aligned}
$$


where $\Delta(x, y)=\left[s_{0}{ }^{2}+s_{1}{ }^{2}+s_{2}{ }^{2}-s_{0} s_{1}-s_{0} s_{2}-\right.$ $\left.s_{1} s_{2}\right]^{1 / 2}$, with $s_{0}, s_{1}$, and $s_{2}$ evaluated at each pixel $(x, y)$ and $\operatorname{sign}(x)=1$ if $x>0,0$ if $x=0$, and -1 if $x<0$. Next we consider the properties of the above CW operation: (a) the transformed components become orthogonal in the sense that their circular autocorrelation function, defined as

$$
R_{k}(x, y)=1 / 3 \sum_{n} \tilde{s}_{n}(x, y) \tilde{s}_{(n-k) \operatorname{modulo3}}(x, y)
$$

is the Kronecker delta function, that is, $R_{k}(x, y)=$ $\delta(k)$; (b) if the color components $\mathrm{R}, \mathrm{G}$, and $\mathrm{B}$ of an

(a)

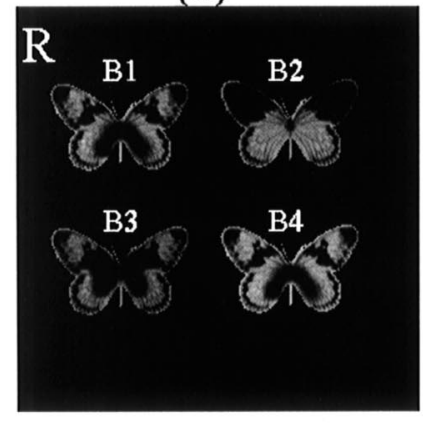

(b)

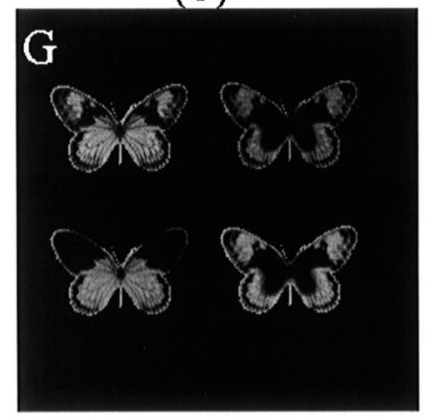

(c)

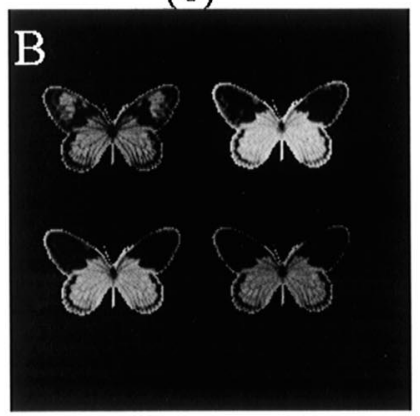

Fig. 1. (a)-(c) Test RGB color scene, (d)-(f) transformed color scene. input signal are nonnegative (real-life images), then the range of the transformed signal $\tilde{R}, \tilde{G}, \tilde{B}$ (from its minimum to its maximum) is $[-1,3]$.

Computer simulation and optical experiments are performed to illustrate the performance of color pattern recognition based on RGB component CW. In our tests a color scene of four butterflies with the same shape but with different distributions of color is used. The test color image is $256 \times 256$ pixels, and the range of the signal in each channel is $[0,1]$. Figure 1 shows the RGB channels corresponding to the color image. The butterfly to be recognized is butterfly B4, following the convention that is shown in Fig. 1(a). We carry out the correlation filtering between original RGB channels and phase-only filters matched to the transmission of butterfly B4 in each channel independently. Summing correlationintensity outputs, we obtain the resulting correlation plane. We call this method direct RGB. Table 1 gives the results for every butterfly.

Figures 1(d)-1(f) show three new images obtained after the whitening procedure described above. The range of these transformed images is $[-1,3]$. The background of the images is equal to zero. Those parts of Figs. 1(d)-1(f) that are darker than the background are negative, and those parts that are brighter than the background are positive. Phaseonly filters have been matched to the transmission of butterfly B4 in each transformed channel independently. Table 1 gives the numerical values of the correlation peaks after the addition of intensities on correlation planes obtained with these three new channels. We refer to this method as circular whitened RGB (CW RGB). One can see an improvement in the recognition process because false alarms are reduced and the discrimination capability is increased very much.

A drawback of using the whitening procedure in optical pattern recognition is the need to introduce a whitened scene with positive and negative values in an optical setup. Different techniques can be applied to solve this problem. Recently, the introduction of the input scene in an optical setup as a phase-encoded image was proposed..$^{9-11}$ We transform a transformed image $f(x, y)$ into a phase-only image $\overline{f(x, y)}$ in the following way:

$$
\overline{f(x, y)}=\exp (i \pi\{[f(x, y)-\min ] /(\max -\min )\}),
$$

where max and min are the maximum and minimum values of $f(x, y)$, respectively. In this way the image $f(x, y)$ has a one-to-one correspondence with the

\begin{tabular}{|c|c|c|c|c|c|c|c|c|}
\hline \multirow[b]{2}{*}{ Method } & \multicolumn{2}{|c|}{ B1 } & \multicolumn{2}{|c|}{ B2 } & \multicolumn{2}{|c|}{ B3 } & \multicolumn{2}{|c|}{ B4 } \\
\hline & CS & EXP & CS & EXP & CS & EXP & $\mathrm{CS}$ & EXP \\
\hline DR RGB & 0.92 & 0.84 & 0.87 & 0.82 & 0.73 & 0.68 & 1 & 1 \\
\hline CW RGB & 0.52 & - & 0.13 & - & 0.08 & - & 1 & - \\
\hline PES RGB & 0.90 & 0.85 & 0.78 & 0.87 & 0.70 & 0.68 & 1 & 1 \\
\hline PES WC & 0.47 & 0.60 & 0.18 & 0.34 & 0.14 & 0.19 & 1 & 1 \\
\hline
\end{tabular}

Table 1. Normalized Correlation Peak Values ${ }^{a}$

${ }^{a} \mathrm{CS}$, computer simulation; EXP, experimental results. 


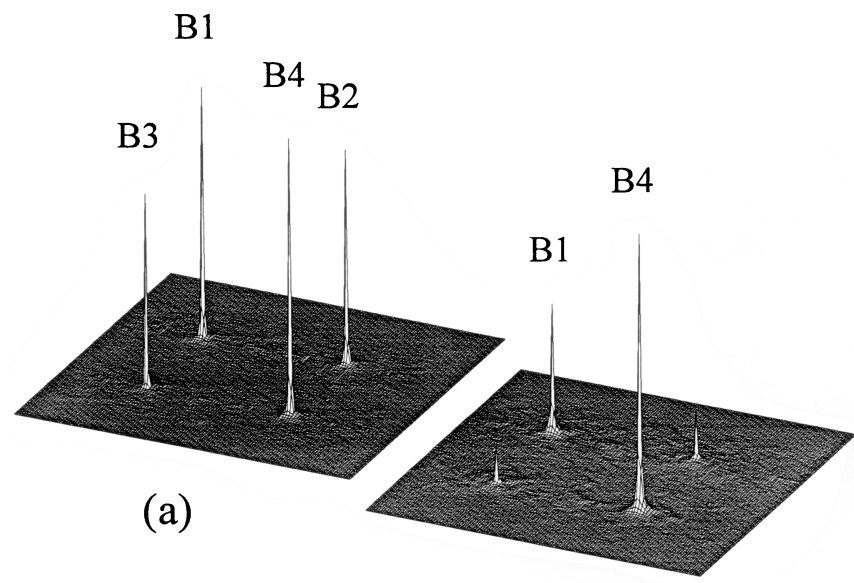

(b)

Fig. 2. Numerical correlation-intensity distributions for (a) the phase-encoded RGB color scene in Figs. 1(a)-(c) and (b) the transformed color scene in Figs. 1(d)-(f).

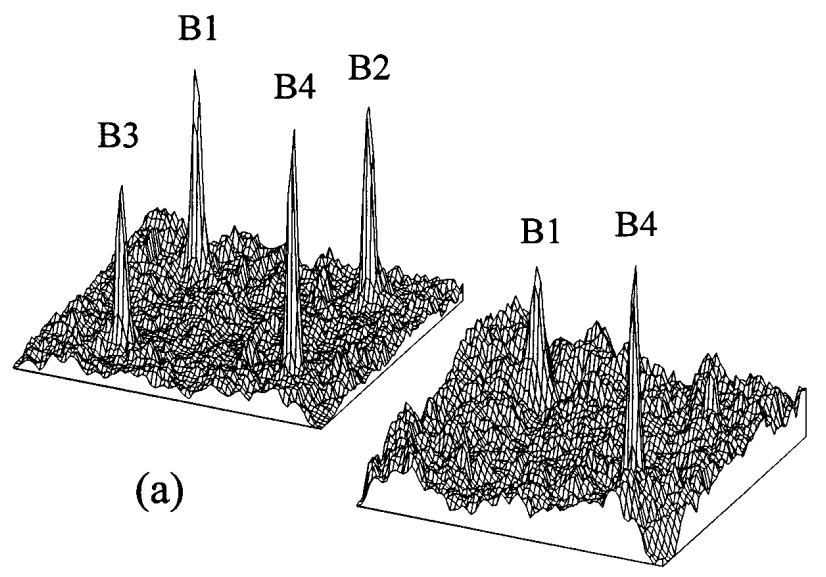

(b)

Fig. 3. Optical correlation-intensity distributions for (a) the phase-encoded RGB color scene in Figs. 1(a)-(c) and (b) the transformed color scene in Figs. 1(d)-(f).

phase-only image $\overline{f(x, y)}$, and moreover we preserve the monotonicity property of $f(x, y)$ in the encoded image $\overline{f(x, y)}$. The latter means that for each three numbers $V_{1}, V_{2}, V_{3}$ in the interval [min, max] with, say, $V_{3}-V_{1}>V_{2}-V_{1}>V_{3}-V_{2}$, the phase-encoding transformation holds relations between the distances of the corresponding points $\overline{V_{1}}, \overline{V_{2}}, \overline{V_{3}}$ on the complex plane, that is, $\left|\mathbf{V}_{3}-\mathbf{V}_{1}\right|>\left|\mathbf{V}_{2}-\mathbf{V}_{1}\right|>\left|\mathbf{V}_{3}-\mathbf{V}_{2}\right|$, where $\mathbf{V}_{i}$ denotes a vector from the origin to the point $\overline{\mathbf{V}_{i}}$ on the complex plane. From the properties of $\mathrm{CW}$ for real-color images we have $\min =-1$ and $\max =3$. In this case, the phase-only filter is the conjugated phase distribution of the Fourier transform of the transformed, phase-encoded target. We call this method the phase-encoded scene with whitened channels (PES WC). Table 1 shows the results of a computer simulation and experimental results. We show that by applying the phase-encoding transformation in Eq. (5) to the transformed images, we obtain results in terms of discrimination capability rather similar to those of the
CW RGB method. To illustrate the improvement that can be achieved with $\mathrm{CW}$ preprocessing, we compare three-dimensional pictures of output correlation planes without and with the whitening (the computer simulation is shown in Fig. 2, and the experimental results in Fig. 3). In both cases the images are used as phaseonly images. We show that four high peaks are obtained in Figs. 2(a) and 3(a), and no recognition can be performed. In contrast, from Figs. 2(b) and 3(b) one can recognize the target with a threshold of $60 \%$. The corresponding normalized correlation intensity peaks are provided in Table 1, in which PES RGB denotes the method with phase representation of the original RGB components. We note that a good agreement with the computer-simulated results is obtained.

The optical experiments have been done with an optical convergent correlator that uses two spatial light modulators. The two modulators were two twisted nematic liquid crystal panels from the Epson VideoProjector VP-100PS. These twisted nematic liquid crystals can be used to perform phase-mostly modulations, so we can use them to introduce the scene as a phase-only image and to introduce the corresponding matched phase-only filter.

In summary, we have applied whitening preprocessing of color components (RGB) to color pattern recognition. Computer simulation and experimental results show that whitening preprocessing of color channels strongly improves recognition performance.

This research has been financed by the Comisión Interministerial de Ciencia y Tecnología, project number TAP93-0667-C03-01, and by Comissió Interdepartamental de Recerca i Innovació Technològica (Generalitat de Catalunya), project number GRQ932047. V. Kober acknowledges financial support from Generalitat de Catalunya. V. Lashin acknowledges financial support from the Ministry of Education and Science of Spain.

\section{References}

1. T. T. S. Yu, Opt. Eng. 23, 690 (1984).

2. E. Badiqué, Y. Komiya, N. Ohyama, J. Tsujiuchi, and T. Honda, Opt. Commun. 61, 181 (1987).

3. M. S. Millán, J. Campos, C. Ferreira, and M. J. Yzuel, Opt. Commun. 73, 277 (1992).

4. M. S. Millán, M. J. Yzuel, J. Campos, and C. Ferreira, Appl. Opt. 31, 2560 (1992).

5. M. J. Yzuel and J. Campos, in Current Trends in Optics, J. C. Dainty, ed. (Academic, San Diego, Calif., 1994), p. 209.

6. J. L. Horner and P. D. Gianino, Appl. Opt. 23, 812 (1984).

7. M. S. Millán, M. Corbalán, J. Romero, and M. J. Yzuel, Opt. Lett. 20, 1722 (1995).

8. L. P. Yaroslavsky, in Progress in Optics, E. Wolf, ed. (Elsevier, New York, 1993), Vol. XXXII, pp. 145-201.

9. S. Mazé and P. Réfrégier, Opt. Lett. 17, 426 (1992).

10. R. R. Kallman and D. H. Goldstein, Opt. Eng. 33, 1806 (1994).

11. K. Styczynski, J. Campos, M. J. Yzuel, and K. Chalasinska-Macukow, Opt. Commun. 118, 193 (1995). 